\title{
Cáncer de mama y embarazo por inseminación artificial en madre con edad materna de riesgo
}

\author{
Breast cancer and pregnancy by artificial insemination \\ in mother with maternal age at risk \\ Kachira Eldadi Portillo Chiu, ${ }^{*}$ César Ramón Aguilar Torres, ${ }^{\ddagger}$ Óscar Salcido Rivera ${ }^{\S}$
}

\begin{abstract}
* Médico residente de cuarto año de Ginecología y Obstetricia. Hospital Central del Estado, Chihuahua, México. ${ }^{\ddagger}$ Médico Ginecólogo. Médico adscrito a la Unidad de Ginecología y Obstetricia del Hospital Central del Estado, Chihuahua, México. § Médico Ginecólogo y Especialista en Biología de la Reproducción. Hospital Star Médica, Chihuahua, México.
\end{abstract}

Correspondencia: Kachira Eldadi Portillo Chiu C. Cosmos 4903 Col. Abraham González, Chihuahua, Chihuahua. E-mail: kachiraportillo@ gmail.com

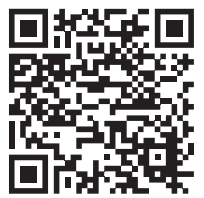

\section{RESUMEN}

Mujer de 42 años con antecedente de madre finada por cáncer de mama; paciente gesta 1, embarazo por inseminación artificial; estimulada por dos ciclos con letrozol y semen de donante, el cual llega con éxito a embarazo, fecha de última menstruación 9 de mayo de 2018. A las nueve semanas de gestación, inicia y detecta masa sólida en mama izquierda dolorosa a la palpación; a la exploración física se encuentra tumoración en mama izquierda en cuadrante inferior externo en el radio de las 4 del reloj, de aproximadamente $5 \mathrm{~cm}$, de consistencia petrosa móvil, irregular y dolorosa a la palpación; cuenta con reporte de sonografía que reportaba proceso tumoral extenso, zona muy vascularizada, vasos gruesos y lesión muy sospechosa de proceso maligno; clasificación BIRADS 4B. Se toma biopsia con aguja de corte y en axila por aspiración con aguja fina que reporta carcinoma ductal invasor moderadamente diferenciado con metástasis a ganglios axilares. Se realiza mastectomía radical modificada izquierda con drenaje axilar a las 17 semanas de gestación, se maneja posteriormente con ocho ciclos de quimioterapia adyuvante, así como 25 ciclos de radioterapia y trastuzumab.

Palabras clave: Cáncer de mama, inseminación artificial, embarazo, reporte de caso.

\section{ABSTRACT}

It is a 42-year-old female patient, mother terminated by breast cancer; patient gesta 1, pregnancy by artificial insemination; date of last menstruation May 9, 2018. Starts at 9 weeks of gestation, detecting the mass as the painful mother on palpation, a Physical examination is on the left, on the bottom, external, on the radio 4 of the watch, approximately $5 \mathrm{~cm}$ of mobile petrous consistency, irregular and painful on palpation; has a sonography report that reports a large vascularized tumor area, thick vessels and very suspicious lesion of malignant process; BIRADS $4 B$ classification; biopsy with cutting needle and axilla by fine needle aspiration that reports moderately differentiated invasive ductal carcinoma with metastasis to axillary lymph nodes. Left modified radical mastectomy with axillary drainage is performed at 17 weeks of gestation and is subsequently managed with 8 cycles of adjuvant chemotherapy, as well as 25 cycles of radiotherapy and trastuzumab.

Keywords: Breast cancer, artificial insemination, pregnancy, case report.

Citar como: Portillo CKE, Aguilar TCR, Salcido RO. Cáncer de mama y embarazo por inseminación artificial en madre con edad materna de riesgo. Rev Mex Mastol. 2020; 10 (1): 18-24. https://dx.doi.org/10.35366/95763 


\section{INTRODUCCIÓN}

El cáncer de mama es una de las entidades oncológicas más frecuentes en las mujeres de nuestro país, aunque esté asociado al embarazo suele tratarse de una asociación rara. Representa el 2.6-6.9\% de todas las neoplasias de mama en las mujeres con edad inferior a los 45 años. Aproximadamente $2 / 3$ de todos los casos ocurren en el postparto y el 1/3 restante durante la gestación, asociándose de forma habitual a un diagnóstico más bien tardío que varía entre los dos y 13 meses. $^{1}$

Los cambios fisiológicos de la glándula mamaria durante la gestación y la lactancia dificultan y retrasan el diagnostico. ${ }^{2}$

Hacemos hincapié en que la edad en el primer embarazo, la ausencia de la lactancia materna y los antecedentes familiares se destacan como posibles factores de riesgo. ${ }^{3}$

El estudio de imagen inicial preferido es el ultrasonido mamario; la mamografía debe solicitarse si hay sospecha de multicentricidad o bilateralidad. El diagnóstico se corrobora con biopsia por aguja de corte. ${ }^{2}$

Al mismo tiempo, hay que tener en cuenta que la incidencia de embarazos a término tras el diagnóstico de cáncer de mama es muy pequeña, alrededor de 3-8\%, y corresponde sólo a $20 \%$ de las pacientes que desearían tener descendencia. Por otra parte, se produce un incremento en el porcentaje de abortos espontáneos (asociándose un riesgo relativo de 1.7 [IC 95\%: 1.1-2.8]). ${ }^{4}$

\section{PRESENTACIÓN DEL CASO}

Mujer de 42 años de edad; originaria y residente de Chihuahua, con antecedente de madre y tía materna finadas por cáncer de mama. Niega hábito tabáquico o consumo de alcohol, sin enfermedades cronicodegenerativas. Antecedente quirúrgico de miomectomía en 2017. Antecedentes ginecológicos y obstétricos: menarca 15 años, inicio de vida sexual activa (IVSA) 21 años, parejas sexuales tres, fecha de última menstruación (FUM) 09/05/2018, fecha probable de parto (FPP) 16/02/2019; gestas una, partos cero, cesárea cero, abortos cero, embarazo con control prenatal con adecuado control prenatal.

Padecimiento actual: paciente inicia las nueve semanas de gestación, detectando pre- sencia de tumoración en mama izquierda, por lo que acude a su valoración al Hospital Central del Estado de Chihuahua.

Se realiza sonografía de mama con reporte de lesiones quísticas en ambas mamas, llamando la atención una gran lesión de contorno irregular, formada por varias lesiones de aspecto nodular, zona muy vascularizada, localizada en el cuadrante inferior interno. Dicha lesión mide $6.4 \times 1.9 \times 4.2 \mathrm{~cm}$, la cual se extiende a región retroareolar hacia la periferia de la mama y axila izquierda; ganglio de $8.4 \mathrm{~mm}$, clasificando como BIRADS 4B.

Se toma biopsia con aguja de corte y en axila por aspiración con aguja fina que reporta carcinoma ductal invasor moderadamente diferenciado, con metástasis a ganglios axilares.

A la exploración física se encuentra tumoración en cuadrante inferior externo en el radio de las 4 del reloj, de aproximadamente $5 \mathrm{~cm}$ de consistencia petrosa móvil, irregular y dolorosa a la palpación.

Contando con 17 semanas de gestación, se realiza mastectomía radical izquierda modificada con técnica de Auchincloss y disección axilar, encontrando tumoración 3 en cuadrante inferior externo de $4 \mathrm{~cm}$, resto de evento quirúrgico sin complicaciones. Sonografía obstétrica posterior a evento quirúrgico reporta placenta corporal posterior, frecuencia cardiaca fetal 142 latidos por minuto, con 16.5 semanas de gestación.

Sonografía de abdomen superior y radiografía de tórax sin datos patológicos.

El análisis histopatológico reporta carcinoma ductal de $4 \mathrm{~cm}$ con tres ganglios metastásicos, clasificando en estadio T2N1M0. Estudio de inmunohistoquímica reporta receptores de estrógenos negativos, receptores de progesterona negativos, HER $23+$ y KI67 20\%.

Se inicia manejo con quimioterapia adyuvante con cuatro ciclos clorhidrato de doxorrubicina (adriamycin) y ciclofosfamida y cuatro ciclos con docetaxel y trastuzumab.

Se realiza cesárea a las 34.5 semanas de gestación por restricción del crecimiento intrauterino, se obtiene producto con Capurro de 36 semanas de gestación con peso aproximado de 1,975 gramos.

Posterior a evento quirúrgico completa ocho ciclos de quimioterapia se agregó manejo 
con trastuzumab y radioterapia adyuvante 25 sesiones.

\section{DISCUSIÓN}

Actualmente, el cáncer de mama asociado al embarazo se define como un caso de cáncer de mama que ocurre durante el embarazo o dentro de un año siguiente al nacimiento, se ha informado que afecta de 1 en 10,000 a 1 en 3,000 embarazos entre las mujeres menores de 45 años; la tasa varía de 2.6 a $6.9 \%$ de los casos. $^{3}$

Aunque la incidencia de cáncer de mama en la mujer premenopáusica está aumentando, la prevalencia general es baja. Sólo $1 \%$ de las mujeres con cáncer de mama son diagnostidas antes de los 30 años, y $6.6 \%$ antes de los 40 años. El cáncer con frecuencia se presenta en etapas avanzadas de la enfermedad, que son probablemente secundarias al hecho de que la detección de rutina no es recomendada en mujeres menores de 40 años. ${ }^{5}$

Fisiopatología del cáncer de mama. El medio hormonal único durante el embarazo, caracterizado por los niveles elevados de estrógeno circulante, progesterona e IGF1, inducen la proliferación de células mamarias y, por lo tanto, podrían iniciar la tumorigénesis o estimular el crecimiento celular, que ya tienen una transformación maligna. ${ }^{3}$

Otro mecanismo que tiene lugar es la tolerancia inmunológica materna para permitir al feto semialogénico crecer dentro. El perfil inmune de las pacientes embarazadas es modificado; esta condición puede ser utilizada por mecanismos protumorigénicos que permiten el desarrollo del cáncer. Por ejemplo, las células inmunosupresoras, como las células T y $B$ reguladoras se han implicado en facilitar la tolerancia inmune y, en última instancia, el escape del cáncer. ${ }^{5}$

Características del cáncer de mama en el embarazo. La edad promedio en el diagnóstico del cáncer de mama asociado al embarazo es entre los 30 y 38 años; alrededor de $75 \%$ de los casos se diagnostican después del parto y 255 durante el embarazo. ${ }^{3}$ El tipo histológico más común es el carcinoma ductal invasor que representa $78-88 \%$ de los casos. ${ }^{3}$ Adenocarcinomas ductales invasivos poco diferenciados fueron prevalentes y con frecuencia receptores de hormonas negativos. ${ }^{6}$

Estos tumores son más grandes, con mayor grado histológico, con invasión vascular y linfática y mayor afectación de los nódulos axilares que en pacientes no embarazadas de la misma edad. ${ }^{5}$

Los tumores de mujeres embarazadas presentan con mayor frecuencia patrones patológicos adversos, tales como receptor de estrógeno (ER) y receptor de progesterona (PR), negatividad y factor de crecimiento epidérmico humano, positividad del receptor 2 (HER2), que está asociada con mayor agresividad del tumor. ${ }^{7}$

\section{Factores de riesgo}

Dentro de los factores de riesgo para cáncer de mama asociado al embarazo, se encuentran los siguientes factores:

Edad. La edad se considera el principal factor de riesgo para desarrollar cáncer de mama en el embarazo, ya que las madres mayores que son primerizas son más propensas a desarrollar cáncer de mama en el embarazo que las mujeres más jóvenes. ${ }^{3} \mathrm{Si}$ las mujeres primíparas tienen más de 35 años, el riesgo elevado puede persistir de por vida. ${ }^{7}$

Historia familiar. Inicialmente, fue sugerido por un estudio de control de casos que las mujeres con la línea germinal asociada la mutación de BRCA1/2 fueron significativamente más propensas a desarrollar cáncer de mama a los 40 años de edad, que las portadoras que eran nulíparas y que el riesgo incrementó con el número de nacimientos. ${ }^{3}$

Lactancia materna. La lactancia materna disminuye de por vida el riesgo de desarrollar cáncer de mama, aunque no necesariamente el cáncer de mama en el embarazo, hasta $4.3 \%$ por cada 12 meses de lactancia materna. ${ }^{3}$

La lactancia materna disminuye el riesgo de cáncer de mama, especialmente de tumores $\mathrm{RH}$ - y Her2+, de manera directamente proporcional a su duración, añadiéndose a la protección del embarazo por sí mismo. ${ }^{4}$

Origen racial. Las tasas de diferentes orígenes raciales tienen distintas tasas de incidencia de cáncer de mama y mortalidad. Hasta la fecha, la relación entre etnicidad y cáncer de mama asociado al embarazo no 
ha sido suficientemente explorada. Un gran estudio de casos y controles basado en el Registro de Cáncer de California, encontró diferencias estadísticamente significativas en etnicidad entre los casos; las mujeres con casos de cáncer de mama asociado al embarazo eran menos propensas a ser blancas y mayor proporción eran hispanas o asiáticas/isleñas del pacífico. ${ }^{3}$

Después de considerar una serie de factores reproductivos, se encontró que sólo el tiempo desde el nacimiento más reciente fue un predictor independiente de mortalidad tras cáncer de mama diagnóstico. Otros factores reproductivos, incluyendo la edad al primer parto, la paridad y la duración de la lactancia materna, no fueron asociados a la mortalidad tras el diagnóstico de cáncer de mama. ${ }^{8}$

Tratamientos de fertilidad como riesgo de cáncer de mama

No existen estudios prospectivos y aleatorizados que demuestren el aumento del cáncer de mama con la utilización de los tratamientos de fertilidad. Algunos estudios introducen variables que modifican el riesgo potencial, aumentándolo en pacientes con riesgo familiar o en nulíparas. ${ }^{4}$ El nivel de recomendación debería ser B, explicando que los estudios epidemiológicos no ven aumentado el riesgo de cáncer de mama con la utilización de tratamientos de fertilidad en pacientes estériles. ${ }^{4}$

No existe evidencia clínica de aumento de riesgo de cáncer de mama en mujeres sanas sometidas a tratamiento de fertilidad. No hay datos específicos para mujeres con alto riesgo de cáncer de mama (riesgo familiar). ${ }^{4}$ Es recomendable realizar una mamografía antes de la técnica de reproducción asistida, especialmente en mujeres mayores de 40 años. ${ }^{4}$

\section{DIAGNÓSTICO}

Las pacientes con cáncer de mama y embarazo suelen ser diagnosticadas con una enfermedad más avanzada. Esto se atribuye principalmente a un retraso en el diagnóstico, se ha informado un retraso en el diagnóstico de cinco a 10 meses, en comparación con uno a cuatro meses en pacientes no embarazadas. ${ }^{9}$
A menudo, un retraso en el diagnóstico de cáncer es secundario al embarazo y la lactancia, debido al incremento en el tamaño y densidad del tejido mamario en este periodo. Con la intención de reducir los retrasos en el diagnóstico, se deben investigar masas palpables que persisten por más de dos semanas durante el embarazo y la lactancia. ${ }^{9}$

Respecto a las pruebas complementarias, se debe realizar una mamografía durante el embarazo, con adecuada protección abdominal. La exposición a la radiación para el feto se estima en 0.4 cGy; sin embargo, la ecografía mamaria es probablemente la mejor técnica para el diagnóstico de cáncer de mama durante el embarazo, por varias razones: es útil para distinguir entre masas mamarias sólidas y quísticas, y también es el método más eficaz para identificar metástasis axilares y no implica ningún riesgo de exposición fetal a la radiación, y también hace posible realizar biopsias percutáneas fácilmente. No hay datos prospectivos sobre la seguridad de la resonancia magnética de mama para diagnosticar masas mamarias en mujeres embarazadas, debido al uso de contraste de gadolinio que podría causar anormalidades. Por lo tanto, esta prueba no se recomienda en esta población. ${ }^{9}$

Es importante tener en cuenta que debe realizarse una biopsia de cualquier masa clínica sospechosa, incluso si la mamografía y la ecografía son poco concluyentes. Si la biopsia confirma la existencia de cáncer de mama, la estatificación inicial debe incluir una historia clínica completa y examen físico; radiografía de tórax con adecuada protección abdominal y una ecografía del hígado. Tomografía computarizada y gammagrafía ósea no se recomiendan debido al riesgo de exposición a la radiación fetal. ${ }^{9}$

Los estudios de extensión sugeridos son: ${ }^{10,11}$

- Radiografía de tórax con protección abdominal. $^{2}$

- Ultrasonido hepático. ${ }^{2}$

- Resonancia magnética de columna toracolumbar sin medio de contraste en caso de sospecha de enfermedad ósea. ${ }^{2}$

- Con información limitada, se ha propuesto resonancia de cuerpo entero durante el segundo y tercer trimestre como una opción a otros estudios de extensión. ${ }^{12}$ 
Tratamiento

Las mujeres diagnosticadas con cáncer de mama en el embarazo deben ser evaluadas por un equipo multidisciplinario que incluye médicos oncólogos, cirujanos de senos, radioterapeutas, ginecólogos de la fertilidad, obstetras, pediatras. ${ }^{9}$

\section{Tratamiento quirúrgico}

El planteamiento quirúrgico recomendado en función del trimestre de gestación seguirá las siguientes pautas.

- Primer y segundo trimestres:

- Mastectomía en los casos que precisen radioterapia $(\mathrm{RT})$ inmediata.

- Se puede optar por cirugía conservadora en los casos en que esté indicada. ${ }^{4}$

Sin embargo, la cirugía en el primer trimestre a menudo se retrasa, muchos cirujanos elegirán esperar hasta después de la semana 12 de gestación, cuando el riesgo de aborto espontáneo disminuye. ${ }^{9}$

- Tercer trimestre:

- Cualquier cirugía es válida. ${ }^{4}$

La cirugía conservadora de mama está indicada en el segundo y tercer trimestres de la gestación, seguida de radioterapia al finalizar el embarazo. ${ }^{2}$

Biopsia selectiva del ganglio centinela (BSGC) en mujeres gestantes. La dosis de radiación expuesta al feto es inferior a la ambiental y no han sido reportados problemas; por lo tanto, se puede realizar advirtiendo a la paciente de la escasa información que tenemos al respecto. Los colorantes están contraindicados. ${ }^{4}$

\section{Tratamiento sistémico}

El tratamiento sistémico del cáncer de mama durante la gestación debe seguir las siguientes recomendaciones:

- Ser manejado por unidades multidisciplinarias con experiencia y formadas por oncólogos, obstetras, radiólogos, cirujanos de mama, radioterapeutas y pediatras neonatólogos. ${ }^{4}$

- Evitar el tratamiento las tres semanas previas al parto por riesgo de sepsis y hemorragia. ${ }^{4}$

- En el segundo y tercer trimestres se puede producir retraso de crecimiento uterino. Ninguna serie ha reportado un número mayor de muertes fetales. ${ }^{4}$

- El tratamiento hormonal está contraindicado durante toda la gestación (Categoría 4 OMS). ${ }^{4}$

- El tratamiento de quimioterapia está contraindicado durante el primer trimestre (Categoría 4 OMS). ${ }^{4}$

- En el segundo y tercer trimestres se recomienda emplear los esquemas en los que hay amplia experiencia, siempre que sea posible. $^{4}$

- Hay amplia experiencia en el empleo seguro de esquemas que incluyen antraciclinas, ciclofosfamida y 5-fluorouracilo (Categoría 1 OMS). Hay menos experiencia para el empleo de taxanos, navelbine y sales de platino. $^{4}$

- Está contraindicado el empleo de trastuzumab (Herceptin) (Categoría 4 OMS). ${ }^{4}$ Ya que se ha asociado a oligoanhidramnios e hipoplasia pulmonar. ${ }^{13-15}$

- Lapatinib no debe emplearse de forma rutinaria (Categoría 3 OMS). Por su mecanismo de acción (inhibición del dominio intracelular de la tirosíncinasa) puede tener efectos sobre el feto. Deberían individualizarse los casos y valorar el riesgobeneficio. $^{4}$

\section{Radioterapia}

- La radioterapia está contraindicada en toda la gestación (Categoría 4 OMS). ${ }^{4}$

- Excepcionalmente, e incrementando las precauciones y la protección fetal, se puede valorar la radioterapia mediante acelerador de electrones, en el segundo y tercer trimestres, para el tratamiento específico de metástasis (Categoría 3 OMS). ${ }^{4}$

\section{Terapia endocrina}

No se recomienda la terapia endocrina durante el embarazo. Se ha demostrado que el tamoxi- 
feno causa defectos de nacimiento, abortos espontáneos y muerte fetal. ${ }^{9}$

Terminación del embarazo

- El tiempo en que debe interrumpirse el embarazo y la vía de terminación de la gestación debe obedecer a indicación obstétrica. $^{2}$

- En caso de recibir quimioterapia, ésta no debe aplicarse tres semanas antes de la fecha probable de parto o después de la semana 35, para evitar NADIR. ${ }^{2}$

- La interrupción del embarazo durante el primer trimestre sólo debe considerase en etapas avanzadas que requieran tratamiento sistémico, por el alto riesgo teratogénico. Esta decisión debe ser tomada por la paciente en conjunto con el grupo multidisciplinario. ${ }^{16}$

\section{Pronóstico}

Al realizar una revisión exhaustiva de la literatura, encontramos datos controvertidos. En algunos artículos se ha demostrado que los cánceres de mama asociados al embarazo tienen un peor pronóstico, mientras que en otros se muestra que las mujeres que estaban embarazadas en el momento del diagnóstico o fueron diagnosticadas dentro de un año después del parto, no tuvieron una tasa de recurrencia locorregional más alta, metástasis a distancia o peor tasa global de supervivencia. ${ }^{9}$

Es importante subrayar dos aspectos de mal pronóstico relacionados con el cáncer de mama asociado al embarazo: el primero es la edad, ya que el cáncer de mama en pacientes jóvenes tiene un peor pronóstico y un diagnóstico tardío que permite que el tumor crezca más tiempo, lo que aumenta el potencial metastásico de la enfermedad. ${ }^{9}$

\section{CONCLUSIONES}

El cáncer de mama asociado al embarazo es una entidad patológica que se presenta en el embarazo y hasta un año posterior al evento obstétrico. A pesar de considerarse una entidad poco común dentro de las patologías del embarazo, si éste sucede en mujeres jóvenes tiene peor pronóstico para las mismas.

Es de suma importancia que, en toda mujer en edad fértil o con factores de riesgo, se realicen estudios de imagen una vez al año para la detección oportuna de cualquier tipo de cáncer de mama; de igual manera, es sumamente importante investigar cualquier masa sospechosa durante el embarazo que dure más de dos semanas.

Hay estudios que demuestran que el uso de fármacos inductores de la ovulación aumenta el riesgo de padecer cáncer de mama durante la gestación en pacientes con factores de riesgo o nulíparas.

El manejo, tanto quirúrgico como coadyuvante del cáncer de mama en la mujer embarazada, tiene que ser un tratamiento individualizado, que se basará en las características personales, dentro de las cuales estará el trimestre en el que se encuentre, así como de la inmuhistoquímica de la tumoración que responda a esta adyuvancia.

El hecho de estar recibiendo tratamiento adyuvante con quimioterapia no es indicación para interrupción del embarazo, a pesar de que este manejo aumenta el riesgo de complicaciones in útero, como restricción del crecimiento intrauterino, sólo amerita una vigilancia más estrecha del mismo.

\section{BIBLIOGRAFÍA}

1. Dudenko D, Hidalgo JJ, Franco A, Lluch A, Cano A. Preservación de la fertilidad de emergencia en paciente con diagnóstico de cáncer de mama durante embarazo: caso clínico. Rev Iberoam Fert Rep Hum. 2019; 36. 27-31.

2. Masson Doyma México S.A. Consenso Mexicano sobre diagnóstico y tratamiento del cáncer mamario. 2017.

3. Ruiz R, Herrero C, Strasser-Weippl K, Touya D, St Louis J, Bukowski A et al. Epidemiology and pathophysiology of pregnancy-associated breast cancer: a review. Breast. 2017; 35: 136-141. doi: 10.1016/j. breast.2017.07.008. Epub 2017 Jul 18.

4. Ara C, Giménez MJ, Cusidó M, Fabregas R, Sociedad Española de Senología y Patología Mamaria, con la colaboración de: Sección de Medicina Perinatal de la SEGO, Sociedad Española de Fertilidad, Sociedad Española de Contracepción. Consenso cáncer de mama y fertilidad. Revista de Senología y Patología Mamaria. 2015; 28 (4): 145-196.

5. Christian N, Gemignani ML. Issues with fertility in young women with breast cancer. Curr Oncol Rep. 2019; 21: 58 
6. Amant F, von Minckwitz G, Han SN, Bontenbal M Ring $A E$, Giermek J et al. Prognosis of women with primary breast cancer diagnosed during pregnancy: results from an international collaborative study. J Clin Oncol. 2013; 31 (20): 2532-2539.

7. Froehlich K, Schmidt A, Heger JI, Al-Kawlani B, Aberl CA, Jeschke $U$ etal. Breast cancer during pregnancy: maternal and fetal outcomes. Eur J Cancer. 2019; 115: 68-78.

8. Whiteman MK, Hillis SD, Curtis KM, McDonald JA, Wingo PA et al. Reproductive history and mortality after breast cancer diagnosis. Obstet Gynecol. 2004; 104 (1): 146-154.

9. Martínez MT, Bermejo B, Hernando C, Gambardella V, Cejalvo JM, Lluch A. Breast cancer in pregnant patients: a review of the literature. Eur J Obstet Gynecol Reprod Biol. 2018; 230: 222-227.

10. Committee Opinion No. 723: Guidelines for diagnostic imaging during pregnancy and lactation. Committee on obstetric practice. Obstet Gynecol. 2017; 130 (4): e210-e216. doi: 10.1097/ AOG.0000000000002355.

11. ACR-SPR Practice Parameter for imaging pregnant or potentially pregnant adolescents and women with ioni- zing radiation. Revised 2018 (Resolution 39). [Consulted 5 December 2018]: https://www.acr. org/-/media/ACR/ Files/Practice-Parameters/Pregnant-Pts.pdf.

12. Peccatori FA, Codacci-Pisanelli G, Del Grande M, Scarfone G, Zugni F, Petralia G. Whole body MRI for systemic staging of breast cancer in pregnant women. Breast. 2017; 35: 177-181. doi: 10.1016/j. breast.2017.07.014.

13. Lambertini M, Peccatori FA, Azim HA Jr. Targeted agents for cancer treatment during pregnancy. Cancer Treat Rev. 2015; 41 (4): 301-309. doi: 10.1016/j.ctrv.2015.03.001.

14. Zagouri F, Sergentanis TN, Chrysikos D, Papadimitriou CA, Dimopoulos MA, Bartsch R. Trastuzumab administration during pregnancy: a systematic review and meta-analysis. Breast Cancer Res Treat. 2013; 137 (2): 349-357. doi: 10.1007/s10549-012-2368-y.

15. Sekar R, Stone PR. Trastuzumab use for metastatic breast cancer in pregnancy. Obstet Gynecol. 2007; 110: 507-510.

16. Yu HH, Cheung PS, Leung RC, Leung TN, Kwan WH. Current management of pregnancy-associated breast cancer. Hong Kong Med J. 2017; 23 (4): 387-394. doi: 10.12809/hkmj166049. 